\title{
Occurrence of a Hybrid Between Taenia saginata and Taenia asiatica Tapeworms in Cambodia
}

\author{
Taehee Chang1', Bong-Kwang Jung1 ${ }^{1}$, Sooji Hong1', Hyejoo Shin', Seungwan Ryoo', Jeonggyu Lee', \\ Keon Hoon Lee', Hansol Park², Keeseon S. Eom², Virak Khieu³, Rekol Huy ${ }^{3}$, Woon-Mok Sohn ${ }^{4}$, \\ Jong-Yil Chai ${ }^{1,5, *}$ (i) \\ 1/nstitute of Parasitic Diseases, Korea Association of Health Promotion, Seoul 07649, Korea; '2Department of Parasitology and Medical Research \\ Institute, Chungbuk National University College of Medicine, Cheongju 28644, Korea; ${ }^{3}$ National Center for Parasitology, Entomology and Malaria \\ Control, Ministry of Health, Phnom Penh, Cambodia; ${ }^{2}$ Department of Parasitology and Tropical Medicine, and Institute of Health Sciences, \\ Gyeongsang National University College of Medicine, Jinju 52727, Korea; ${ }^{5}$ Department of Tropical Medicine and Parasitology, Seoul National \\ University College of Medicine, Seoul 03080, Korea
}

\begin{abstract}
Human infection with Taenia asiatica or a hybrid between Taenia saginata and T. asiatica has not been reported in Cambodia. We detected for the first time a hybrid form between T. saginata and T. asiatica in Preah Vihear Province, Cambodia. An adult tapeworm specimen, i.e., $75 \mathrm{~cm}$ long strobila without scolex, was expelled from a 27-year-old man after praziquantel medication and purging. It was morphologically indistinguishable between $T$. saginata and $T$. asiatica. Several proglottids were molecularly analyzed to confirm the tapeworm species. The mitochondrial gene encoding cytochrome $c$ oxidase subunit 1 (cox1) and nuclear genes encoding elongation factor-1a (ef1) and ezrin-radixin-moesin (ERM)-like protein (elp) were sequenced, and a single-allele analysis was performed to confirm the haploid genotype. The results revealed that our sample showed a discrepancy between the mitochondrial and 2 nuclear genes. It possessed homozygous sequences typical of $T$. saginata at cox 1 and ef1 loci. However, it was heterozygous at the elp locus, with 1 allele in T. asiatica $(e / p A)$ and 1 in T. saginata $(e / p C)$, which indicates that it is a hybrid between $T$. saginata and T. asiatica. The present results confirmed the presence of a hybrid between $T$. saginata and T. asiatica in Cambodia and strongly suggest the existence of also 'pure' T. asiatica in Cambodia.
\end{abstract}

Key words: Taenia saginata, Taenia asiatica, hybrid, allelic study, Cambodia

Taenia solium, Taenia saginata, and Taenia asiatica are 3 tapeworm species that can cause human intestinal infections. They have unique life cycles, taking humans as the only definitive host and pigs or cattle as the intermediate host [1]. T. asiatica, which is transmitted by swine (viscera), was first identified in Taiwan and then in South Korea and reported as a distinct species from T. saginata in 1993 [1,2]. The distribution of human taeniases by T. solium and T. saginata is worldwide, whereas $T$. asiatica is found mostly in Asian countries [3,4].

The possibility of hybridization between $T$. asiatica and $T$. saginata has been raised in previous studies that analyzed samples from several countries, including Thailand, China, the Philippines, and Lao PDR [5-8]. Although the 2 taxa have dis-

\footnotetext{
- Received 16 February 2021, revised 17 March 2021, accepted 17 March 2021.

*Corresponding author (cjy@snu.ac.kr)

(C) 2021. Korean Society for Parasitology and Tropical Medicine

This is an Open Access article distributed under the terms of the Creative Commons Attribution Non-Commercial License (https://creativecommons.org/licenses/by-nc/4.0) which permits unrestricted non-commercial use, distribution, and reproduction in any medium, provided the original work is properly cited.
}

tinct biological characteristics, including host specificity, gene exchange between the 2 species was strongly suggested using allelic analysis on mitochondrial and nuclear genetic markers [5]. The existence of T. solium and T. saginata in Cambodia was confirmed by DNA sequencing of egg-positive fecal samples and adult worms expelled from residents $[9,10]$. However, there has been no report on the occurrence of T. asiatica or a hybrid between T. saginata and T. asiatica in humans despite its geographical location, surrounded by other countries where $T$. asiatica and hybrids between the 2 species have been found [5$8,11]$. In this study, we report a hybrid individual between $T$. saginata and T. asiatica in a northern part of Cambodia, bordering Lao PDR, suggesting the occurrence of T. asiatica in this country.

Fecal samples were collected from total 1,156 people in 2 northern provinces (5 villages each), Preah Vihear and Stung Treng (Fig. 1), and examined by the Kato-Katz thick smear technique, as previously reported [10]. The fecal survey of people and worm collection after praziquantel medication were 




Fig. 1. Map of the areas for fecal surveys in Cambodia (A, Preah Vihear Province; B, Stung Treng Province) and the village (red, quadrangle) where the present hybrid case between Taenia saginata and Taenia asiatica was found.

ethically approved by National Ethics Committee for Health Research, Cambodia (IRB no. 099NECHR). Twenty-six (2.4\%) out of them were positive for Taenia spp. eggs, and 3 tapeworm strobilae were collected from 3 patients in Preah Vihear after praziquantel medication (15 mg/kg single dose) and purging with $\mathrm{MgSO}_{4}$.

Two of the 3 strobilae were diagnosed molecularly as 'pure' T. saginata based on the mitochondrial gene encoding cytochrome c oxidase subunit 1 ( $\operatorname{cox} 1$ ), and 2 nuclear genes encoding elongation factor-1a (ef1) and ezrin-radixin-moesin (ERM)-like protein (elp) which were reported previously [10]. The remaining 1 strobila $(75 \mathrm{~cm}$ in length, mostly gravid proglottids without a scolex) expelled from a 27-year-old man showed strange results that the elp allele type was different from 'pure' T. saginata. In this study, we further analyzed the allele type of the elp gene of this strobila. The sample preparation and genomic DNA extraction were conducted as described in our previous study [10]. The analyses of mitochondrial cox 1 (1,620 bp) and 2 nuclear genes ef1 (1,090 bp) and elp (1,160 bp) were performed following conditions of Okamoto et al. [5]. A consistent sequence data of elp locus could not be obtained since double peaks were observed in electro- pherograms. Thus, to confirm the haploid genotype of elp locus, PCR amplification and sequencing were conducted at the single-allele level using T-Blunt PCR cloning kit (Solgent, Seoul, Korea).

Multiple sequences alignment was constructed with Clustal W [12]. A phylogenetic tree of elp locus sequences identified in this study and representative sequences of Taenia spp. available in the GenBank database were constructed using the maximum-likelihood (ML) method based on the Tamura-Nei model of nucleotide substitution. The tree was viewed by MEGA v6 [13]. Bootstrap values were calculated with 1,000 replications.

Our Taenia strobila sample showed a discrepancy in the diagnosis between the mitochondrial and nuclear genes. It possessed homozygous sequences typical of $T$. saginata cox 1 (MT074050) and ef1 (MT075318) loci. However, at the elp locus, it was heterozygous with 1 allele (MT501760) matched with T. asiatica (elpA) and another (MT501761) with T. saginata (elpC) (Table 1; Fig. 2).

The homology between our allele sample elpA and T. asiatica (AB462829-AB462830) was 99.9-100\%, whereas the homology between our sample elpA and T. saginata (AB462841AB462842) or T. solium (AB505026) was lower than that, 99.1- 


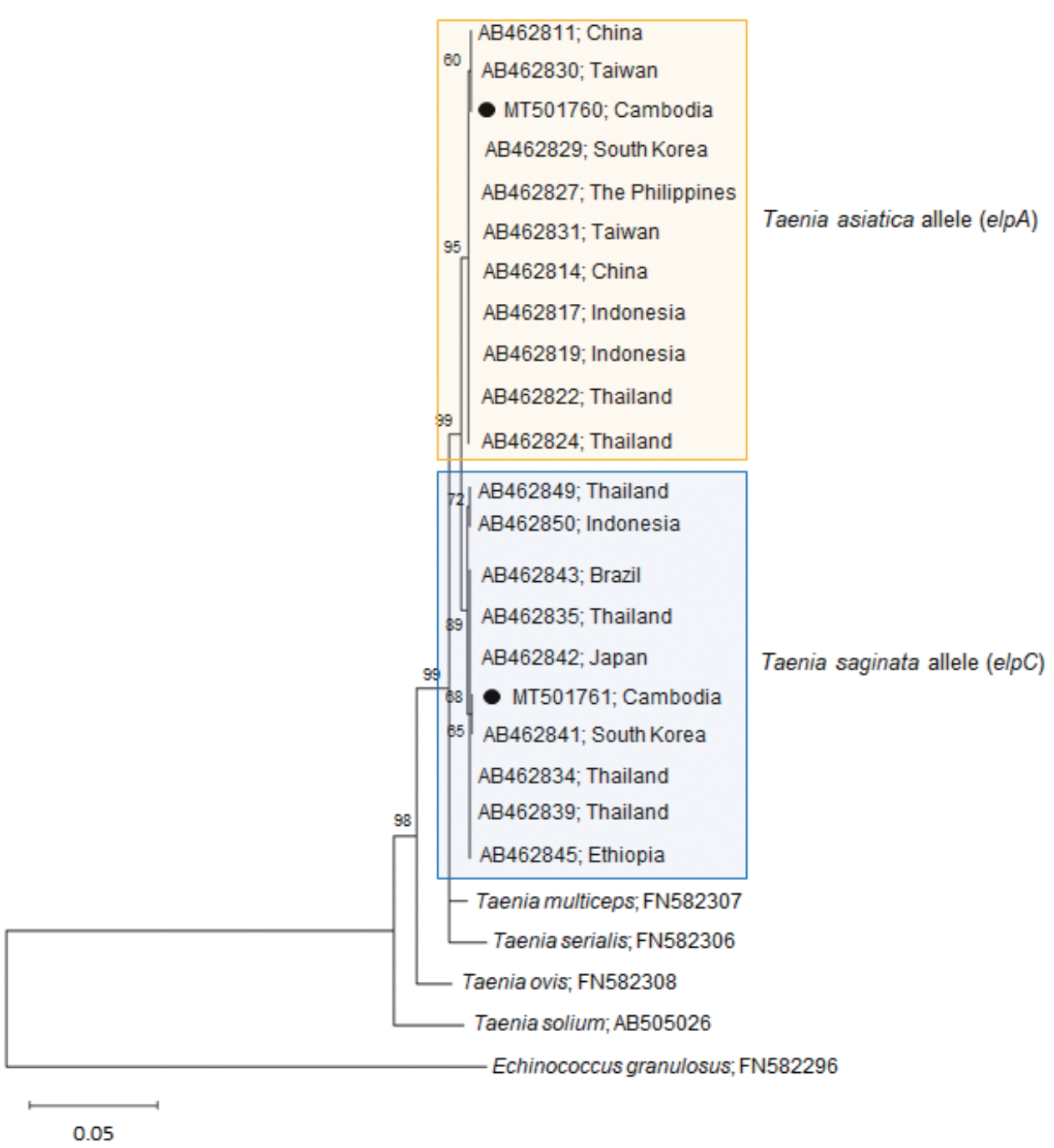

Fig. 2. A phylogenetic tree of our sample (alleles) in comparison with related Taenia spp. tapeworms drawn with elp DNA sequences using the maximum-likelihood method employing Tamura-Nei model of nucleotide substitution with 1,000 bootstrap replications. Black dots (-) indicate the sequences identified in this study. The allele MT501760 has been registered under the name T. saginata in GenBank; however, this allele is actually classified as a T. asiatica allele. Scale bar indicates nucleotide substitutions per site.

Table 1. Genotypes of the Taenia tapeworm sample from our patient

\begin{tabular}{|c|c|c|c|}
\hline \multirow{2}{*}{ Patient } & \multirow{2}{*}{ Mitochondrial gene (cox1) } & \multicolumn{2}{|c|}{ Nuclear genes } \\
\hline & & Genotype at ef1 locus ${ }^{a}$ & Genotype at elp locus ${ }^{a}$ \\
\hline 27-year-old male & T. saginata type & ef1C/ef1C (T. saginata) & elpA/elpC (T. asiatica/T. saginata hybrid) \\
\hline
\end{tabular}

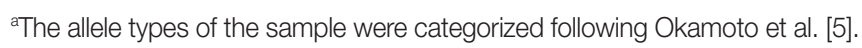

$99.2 \%$ or $94.7 \%$, respectively. In addition, the homology between our allele sample elpC and T. saginata (AB462841AB462842) was 99.9-100\%, whereas the homology between our sample elpC and T. asiatica (AB462829-AB462830) or T. solium (AB505026) was lower than that, $99.1-99.2 \%$ or $94.8 \%$, respectively. These results strongly suggest that our sample is a hybrid between the 2 Taenia species.
Hybridization between $T$. saginata and $T$. asiatica was reported recently in Asian countries [5-8] but not yet in Cambodia. In this study, we performed an allelic analysis of a Taenia tapeworm strobila collected from a human to rule out the hybrid issue in Cambodia and confirmed that our specimen is a hybrid between T. saginata and T. asiatica.

Human taeniases (by T. solium, T. asiatica, and T. saginata) 
are known to be endemic in East and Southeast Asia, including Thailand, Vietnam, Lao PDR, which neighbor with Cambodia, and also in Nepal and India $[8,11,14]$. However, in Cambodia, reports of human taeniasis due to T. asiatica have not yet been documented. Our present finding implies the possible existence of T. asiatica in the northern part of Cambodia, although it remains to be confirmed by further studies.

The transmission of tapeworms is closely related to the lifestyle and cultural characteristics of people, which involve the consumption of undercooked meat infected with viable metacestodes (i.e., cysticerci) [14]. In Cambodia, there is a traditional food habit of eating cattle or pig meat, for example raw beef salad ('pleah sach ko'). However, as the intermediate host and life cycle of the hybrid between T. saginata and T. asiatica are unclear now, it is necessary to examine the metacestodes from domestic animals using genetic markers. Thus, the risk factors as well as the level of exposure of the people in Cambodia to the agents causing human taeniases, including the hybrid, should be evaluated.

\section{ACKNOWLEDGMENTS}

We appreciate the staff in the National Center for Parasitology, Entomology and Malaria Control, Ministry of Health, Phnom Penh, Cambodia, who helped this survey. This work was supported by the Institute of Parasitic Diseases, Korea Association of Health Promotion, Seoul, South Korea.

\section{CONFLICT OF INTEREST}

The authors declare no competing interests related to this study.

\section{REFERENCES}

1. Chai JY. Human taeniasis in the Republic of Korea: Hidden or gone? Korean J Parasitol 2013; 51: 9-17. https://doi.org/10.3347/ kjp.2013.51.1.9

2. Eom KS, Rim HJ. Morphological descriptions of Taenia asiatica sp. n. Korean J Parasitol 1993; 31: 1-6. https://doi.org/10.3347/ kjp.1993.31.1.1

3. Eom KS, Rim HJ. Geographical distribution of Taenia asiatica and related species. Korean J Parasitol 2009; 47 (suppl): 115-124. https:// doi.org/10.3347/kjp.2009.47.S.S115
4. Eom KS, Rim HJ, Jeon HK. Taenia asiatica: historical overview of taeniasis and cysticercosis with molecular characterization. Adv Parasitol 2020; 108: 133-173. https://doi.org/10.1016/bs.apar.2019.12. 004

5. Okamoto M, Nakao M, Blair D, Anantaphruti MT, Waikagul J, Ito A. Evidence of hybridization between Taenia saginata and Taenia asiatica. Parasitol Int 2010; 59: 70-74. https://doi.org/10. 1016/j.parint.2009.10.007

6. Yamane K, Suzuki Y, Tachi E, Li T, Chen X, Nakao M, Nkouawa A, Yanagida T, Sako Y, Sato H, Okamoto M. Recent hybridization between Taenia asiatica and Taenia saginata. Parasitol Int 2012; 61: 351-355. https://doi.org/10.1016/j.parint.2012.01.005

7. Yamane K, Yanagida T, Li T, Chen X, Dekumyoy P, Waikagul J, Nkouawa A, Nakao M, Sako Y, Ito A, Sato H, Okamoto M. Genotypic relationship between Taenia saginata, Taenia asiatica and their hybrids. Parasitology 2013; 140: 1595-1601. https://doi.org/10.1017/ S0031182013001273

8. Sato MO, Sato M, Yanagida T, Waikagul J, Pongvongsa T, Sako Y, Sanguankiat S, Yoonuan T, Kounnavang S, Kawai S, Ito A, Okamoto M, Moji K. Taenia solium, Taenia saginata, Taenia asiatica, their hybrids and other helminthic infections occurring in a neglected tropical diseases' highly endemic area in Lao PDR. PLoS Negl Trop Dis 2018; 12: e0006260. https://doi.org/10.1371/journal. pntd.0006260

9. Jeon HK, Yong TS, Sohn WM, Chai JY, Hong SJ, Han ET, Jeoung HG, Chhakda T, Sinuon M, Socheat D, Eom KS. Molecular identification of Taenia tapeworms by cox1 gene in Koh Kong, Cambodia. Korean J Parasitol 2011; 49: 195-197. https://doi.org/10.3347/ kjp.2011.49.2.195

10. Chang T, Jung BK, Sohn WM, Hong S, Shin H, Ryoo S, Lee J, Lee KH, Khieu V, Huy R, Chai JY. Molecular diagnosis of Taenia saginata tapeworms from two residents of Northern Cambodia. Korean J Parasitol 2020; 58: 201-204. https://doi.org/10.3347/kjp.2020.58.2.201

11. Braae UC, Hung NM, Satrija F, Khieu V, Zhou XN, Wilingham AL. Porcine cysticercosis (Taenia solium and Taenia asiatica): mapping occurrence and areas potentially at risk in East and Southeast Asia. Parasit Vectors 2018; 11: 613. https://doi.org/10.1186/s13071-0183203-Z

12. Larkin MA, Blackshields G, Brown NP, Chenna R, McGettigan PA, McWilliam H, Valentin F, Wallace IM, Wilm A, Lopez R, Thompson JD, Gibson TJ, Higgins DG. Clustal W and Clustal X version 2.0. Bioinformatics 2007; 23: 2947-2948. https://doi. org/10.1093/bioinformatics/btm404

13. Tamura K, Stecher G, Peterson D, Filipski A, Kumar S. MEGA6: Molecular evolutionary genetics analysis version 6.0. Mol Biol Evol 2013; 30: 2725-2729. https://doi.org/10.1093/molbev/mst197

14. Ito A, Li T, Wandra T, Dekumyoy P, Yanagida T, Okamoto M, Budke CM. Taeniasis and cysticercosis in Asia: a review with emphasis on molecular approaches and local lifestyles. Acta Trop 2019; 198: 105075. https://doi.org/10.1016/j.actatropica.2019.105075 